\title{
Hypomagnesemia, CTCAE
}

National Cancer Institute

\section{Source}

National Cancer Institute. Hypomagnesemia, CT CAE. NCI Thesaurus. Code C143570.

A disorder characterized by laboratory test results that indicate a low concentration of magnesium in the blood. 